\title{
Solução ELTAN para o Problema de Transporte com Fonte
}

A.V. CARDONA, ${ }^{1}$ Faculdade de Matemática, Pontifícia Universidade Católica do Rio Grande do Sul, Av. Ipiranga, 6681, Prédio 15, 90619-900, Porto Alegre, RS, Brasil

J.V.P. de OLIVEIRA, ${ }^{2}$ Departamento de Matemática, Universidade Federal de Santa Maria, Av. Roraima, s/n, Prédio 13, 97105-900, Santa Maria - RS - Brasil

Resumo. Recentemente foi apresentada uma nova versão do método LTAN para resolver problemas de transporte lineares homogêneos em uma placa. Esta formulação foi denominada método ELTA $_{N}$ e é baseada na diagonalização de uma matriz $\mathrm{N} \times \mathrm{N}$, em oposição a matriz $(2 \mathrm{~N}) \times(2 \mathrm{~N})$ do método $\mathrm{LTA}_{\mathrm{N}}$ original. Neste trabalho estendemos essa formulação para resolver problemas de transporte não homogêneos. Simulações numéricas são apresentadas e os resultados são comparados aos resultados obtidos pelos métodos $\mathrm{LTS}_{\mathrm{N}}$ e $\mathrm{LTA}_{\mathrm{N}}$.

\section{Introdução}

O método $A_{N}$ foi proposto por Coppa e Ravetto [11] como uma aproximação da forma integral da equação mono-energética de transporte de Boltzmann [16], considerando espalhamento isotrópico e consiste em reduzir essa equação a um sistema de equações diferenciais de segunda ordem na variável espacial, as quais são denominadas equações $A_{N}$. Alternativamente, as equações $A_{N}$ [12] foram obtidas da equação integro-diferencial linear de Boltzmann [16] através da aplicação da transformada de Fourier sobre a variável espacial do fluxo angular de nêutrons. Posteriormente, o método $A_{N}$ foi estendido para o caso de espalhamento anisotrópico [13], com a devida demonstração da convergência, bem como se demonstrou a sua equivalência com os métodos $\mathrm{P}_{2 \mathrm{~N}-1}[14], \mathrm{S}_{2 \mathrm{~N}}[15]$ e $\mathrm{SP}_{2 \mathrm{~N}-1}[10]$.

Posteriormente, Cardona e Vilhena $[3,7,8]$ apresentaram uma nova derivação para as equações $\mathrm{A}_{\mathrm{N}}$ em uma placa, considerando um grupo de energia e anisotropia de grau arbitrário. Estas equações foram obtidas aplicando-se a transformação de Kuznetsov [23] no fluxo angular de nêutrons e utilizando-se a idéia do método $S_{N}$ [22]. A transformação de Kuznetsov consiste em decompor o fluxo angular em duas funções dependentes da variável $\mu \in[0,1] \mathrm{e}$, conseqüentemente, decompor a equação de transporte em duas equações íntegro-diferenciais acopladas nestas funções. Aqui, as equações $A_{N}$ são obtidas aproximando o termo integral das equações resultantes

\footnotetext{
${ }^{1}$ acardona@pucrs.br

2 josepol@smail.ufsm.br
} 
por quadratura de Gauss e usando-se o método da colocação. Nestes trabalhos, também foi proposta a solução destas equações pelo método LTA ${ }_{N}$. Esse método consiste na aplicação da transformada de Laplace nas equações $A_{N}$, resolução da equação algébrica resultante e inversão do fluxo transformado pelo método de Trzaska [28]. O método $\mathrm{LTA}_{\mathrm{N}}$ foi apresentado como uma opção ao método $\mathrm{LTS}_{\mathrm{N}}$ $[29,30]$, mas como se conseguia resolver no máximo as equações $A_{8}$ e, como para estes valores não se encontrou vantagem numérica em relação ao método $\operatorname{LTS}_{16}[6]$, de formulação mais simples, optou-se por abandonar temporariamente esta metodologia.

Baseados nos avanços obtidos pela formulação $\operatorname{LTS}_{N}[18,19,26,27]$, nos quais foram superadas as limitações computacionais para trabalhar com problemas de transporte não-homogêneos que exigem altas ordens de quadratura, em trabalhos recentes, Cardona et al. [4, 9] propuseram uma formulação melhorada do método $\mathrm{LTA}_{\mathrm{N}}$, extremamente robusta do ponto de vista computacional, pois permite trabalhar com problemas de transporte com uma fonte arbitrária e com altas ordens de quadratura ( $\mathrm{N}$ variando até 650). Seguindo a idéia do método $\mathrm{LTS}_{\mathrm{N}}$, este objetivo foi alcançado através da diagonização da matriz $\mathrm{LTA}_{\mathrm{N}}$, a qual é uma matriz $(2 \mathrm{~N}) \times(2 \mathrm{~N})$ resultante da aplicação da transformada de Laplace no conjunto das equações $A_{N}$. Nestes trabalhos, a formulação $L_{T A}$ foi comparada com o método LTS $_{N}$ na resolução de problemas benchmark encontrados na literatura [21], sendo que se observou uma maior velocidade de convergência desta formulação em relação ao método $\mathrm{LTS}_{\mathrm{N}}$. Esta maior taxa de convergência deve ser confirmada e o caminho para tal reside no estudo da convergência da formulação $\mathrm{LTA}_{\mathrm{N}}$, da mesma maneira que foi feito para a formulação $\operatorname{LTS}_{\mathrm{N}}[24,25]$.

Visando reduzir o tempo de computação da formulação $\mathrm{LTA}_{\mathrm{N}}$, recentemente foi proposta uma nova versão deste metódo para resolução de problemas homogêneos de transporte, denominada ELTA $\mathrm{N}_{\mathrm{N}}$ [5]. Esta nova formulação é baseada na inversão do fluxo angular transformado através da diagonalização de uma matriz $\mathrm{N} \times \mathrm{N}$, a qual é obtida após algumas manipulações algébricas sobre as equações $A_{N}$ transformadas. Neste trabalho, essa formulação é estendida para resolução de problemas de transporte não-homogêneos, no nosso caso, problemas dotados de uma fonte.

\section{As Equações $A_{N}$}

Inicialmente discutiremos a derivação das equações $A_{N}$ através da aplicação da transformação de Kuznetsov [23]. Para tanto, consideremos a equação de transporte [16]:

$$
\begin{aligned}
& \mu \frac{\partial \varphi}{\partial x}(x, \mu)+\sigma_{t} \varphi(x, \mu)= \\
& \frac{\sigma_{s}}{2} \sum_{k=0}^{L} \beta_{k} \mathrm{P}_{k}(\mu) \int_{-1}^{1} \mathrm{P}_{k}\left(\mu^{\prime}\right) \varphi\left(x, \mu^{\prime}\right) d \mu^{\prime}+\mathrm{S}(x, \mu), 0 \leq x \leq a,
\end{aligned}
$$

sujeita as seguintes condições de contorno:

$$
\varphi(0, \mu)=f(\mu), \text { se } \mu>0,
$$


e

$$
\varphi(a,-\mu)=0, \text { se } \mu>0,
$$

onde $\varphi(x, \mu)$ denota o fluxo angular, $\mathrm{S}(x, \mu)$ o termo de fonte, $\mathrm{P}_{k}(\mu)$ os polinômios de Legendre e L o grau de anisotropia.

Aplicando a transformação de Kuznetsov, respectivamente, para o fluxo angular e termo de fonte,

$$
\varphi(x, \mu)=\left\{\begin{array}{l}
u(x, \mu)+v(x, \mu), \text { se } \mu>0 \\
u(x,-\mu)-v(x,-\mu), \text { se } \mu<0
\end{array}\right.
$$

e

$$
\mathrm{S}(x, \mu)=\left\{\begin{array}{l}
\mathrm{S}_{\mathrm{P}}(x, \mu)+\mathrm{S}_{\mathrm{I}}(x, \mu), \text { se } \mu>0 \\
\mathrm{~S}_{\mathrm{P}}(x,-\mu)-\mathrm{S}_{\mathrm{I}}(x,-\mu), \text { se } \mu<0
\end{array}\right.
$$

substituindo na equação (2.1) e fazendo algumas manipulações algébricas, obtemos

$$
\begin{aligned}
& \mu \frac{\partial v}{\partial x}(x, \mu)+\sigma_{t} u(x, \mu)= \\
& \sigma_{s} \sum_{k=0}^{\mathrm{L}} \beta_{k} \mathrm{P}_{k}(\mu) \int_{0}^{1} \mathrm{P}_{k}\left(\mu^{\prime}\right) u\left(x, \mu^{\prime}\right) d \mu^{\prime}+\mathrm{S}_{\mathrm{P}}(x, \mu), \mu>0
\end{aligned}
$$

e

$$
\begin{aligned}
& \mu \frac{\partial u}{\partial x}(x, \mu)+\sigma_{t} v(x, \mu)= \\
& \sigma_{s} \sum_{k=0}^{\mathrm{L}} \beta_{k} \mathrm{P}_{k}(\mu) \int_{0}^{1} \mathrm{P}_{k}\left(\mu^{\prime}\right) v\left(x, \mu^{\prime}\right) d \mu^{\prime}+\mathrm{S}_{\mathrm{I}}(x, \mu), \mu>0
\end{aligned}
$$

Então, aplicando o método da colocação na variável angular e aproximando as integrais que aparecem no lado direito das equações (2.6-2.7) por um esquema de quadratura gaussiana, obtemos:

$$
\begin{aligned}
& \mu_{n} \frac{d v}{d x}\left(x, \mu_{n}\right)+\sigma_{t} u\left(x, \mu_{n}\right)= \\
& \sigma_{s} \sum_{k=0}^{\mathrm{L}} \beta_{k} \mathrm{P}_{k}\left(\mu_{n}\right) \sum_{m=1}^{\mathrm{N}} \omega_{m} \mathrm{P}_{k}\left(\mu_{m}\right) u\left(x, \mu_{m}\right)+\mathrm{S}_{\mathrm{P}}\left(x, \mu_{n}\right)
\end{aligned}
$$

e

$$
\begin{aligned}
& \mu_{n} \frac{d u}{d x}\left(x, \mu_{n}\right)+\sigma_{t} v\left(x, \mu_{n}\right)= \\
& \sigma_{s} \sum_{k=0}^{\mathrm{L}} \beta_{k} \mathrm{P}_{k}\left(\mu_{n}\right) \sum_{m=1}^{\mathrm{N}} \omega_{m} \mathrm{P}_{k}\left(\mu_{m}\right) v\left(x, \mu_{m}\right)+\mathrm{S}_{\mathrm{I}}\left(x, \mu_{n}\right) \text {, } \\
& \text { impar }
\end{aligned}
$$

para $n=1: \mathrm{N}(\mathrm{N} \geq 2)$, as quais são conhecidas como equações $A_{\mathrm{N}}$ de transporte. Aqui, $\mu_{k}$ e $\omega_{k}$ denotam, respectivamente, as raízes e os pesos da quadratura de Gauss para o domínio $\mu \in[0,1]$. 


\section{O Método ELTA $_{N}$}

Para obter a nova formulação $\mathrm{LTA}_{\mathrm{N}}$, denominada $\mathrm{ELTA}_{\mathrm{N}}$, inicialmente reescrevemos as equações (2.8-2.9) na forma matricial e aplicamos a transformada de Laplace na variável espacial, resultando:

$$
s \bar{u}(s)=u(0)+\mathrm{B} \bar{v}(s)+\overline{\mathrm{S}}_{\mathrm{I}}(s)
$$

e

$$
s \bar{v}(s)=v(0)+\mathrm{C} \bar{u}(s)+\overline{\mathrm{S}}_{\mathrm{P}}(s),
$$

onde os vetores $u(x), v(x), \mathrm{S}_{\mathrm{I}}(x)$ e $\mathrm{S}_{\mathrm{P}}(x)$ são respectivamente definidos como

$$
\begin{gathered}
{\left[u\left(x, \mu_{1}\right) \ldots u\left(x, \mu_{\mathrm{N}}\right)\right]^{\mathrm{T}},\left[v\left(x, \mu_{1}\right) \ldots v\left(x, \mu_{\mathrm{N}}\right)\right]^{\mathrm{T}}} \\
{\left[\frac{\mathrm{S}_{\mathrm{I}}\left(x, \mu_{1}\right)}{\mu_{1}} \ldots \frac{\mathrm{S}_{\mathrm{I}}\left(x, \mu_{\mathrm{N}}\right)}{\mu_{\mathrm{N}}}\right]^{\mathrm{T}} \mathrm{e}\left[\frac{\mathrm{S}_{\mathrm{P}}\left(x, \mu_{1}\right)}{\mu_{1}} \ldots \frac{\mathrm{S}_{\mathrm{P}}\left(x, \mu_{\mathrm{N}}\right)}{\mu_{\mathrm{N}}}\right]^{\mathrm{T}}}
\end{gathered}
$$

A barra denota a transformada de Laplace destes vetores, I é a matriz identidade $\mathrm{N} \times \mathrm{N}$ e as matrizes $\mathrm{N} \times \mathrm{N}$ B e $\mathrm{C}$ têm, respectivamente, os coeficientes:

$$
\mathrm{B}_{i, j}=\frac{\sigma_{s}}{\mu_{i}} \sum_{\substack{k=0 \\ \text { impar }}}^{L} \beta_{k} \mathrm{P}_{k}\left(\mu_{i}\right) \omega_{j} \mathrm{P}_{k}\left(\mu_{j}\right)-\frac{\sigma_{t}}{\mu_{i}} \delta_{i, j}
$$

e

$$
\mathrm{C}_{i, j}=\frac{\sigma_{s}}{\mu_{i}} \sum_{k=0}^{L} \beta_{k} \mathrm{P}_{k}\left(\mu_{i}\right) \omega_{j} \mathrm{P}_{k}\left(\mu_{j}\right)-\frac{\sigma_{t}}{\mu_{i}} \delta_{i, j} .
$$

Então, substituindo a equação (3.2) em (3.1), depois de algumas manipulações algébricas, obtemos:

$$
\left(s^{2} \mathrm{I}-\mathrm{BC}\right) \bar{u}(s)=s u(0)+\mathrm{B} v(0)+\mathrm{B}_{\mathrm{P}}(s)+s \overline{\mathrm{S}}_{\mathrm{I}}(s) .
$$

Finalmente, fazendo $\mathrm{BC}=\mathrm{XDX}^{-1}$, onde $\mathrm{X}$ é a matriz dos autovetores e D é a matriz diagonal contendo os autovalores de $\mathrm{BC}$ e, utilizando propriedades da transformada de Laplace, após a solução da equação (3.5) e inversão da transformada de Laplace, obtemos:

$$
\begin{aligned}
u(x)=\mathrm{X} & \left(\mathrm{e}^{\mathrm{D}^{\frac{1}{2}}(x-a)} \Delta+\mathrm{e}^{-\mathrm{D}^{\frac{1}{2}} x} \Gamma+\int_{a}^{x} \mathrm{e}^{\mathrm{D}^{\frac{1}{2}}(x-\tau)} \mathrm{F}(\tau) d \tau\right. \\
& \left.+\int_{0}^{x} \mathrm{e}^{-\mathrm{D}^{\frac{1}{2}}(x-\tau)} \mathrm{G}(\tau) d \tau\right)
\end{aligned}
$$


e

$$
\begin{aligned}
v(x)=\operatorname{CXD}^{\frac{1}{2}} & \left(\mathrm{e}^{\mathrm{D}^{\frac{1}{2}}(x-a)} \triangle-\mathrm{e}^{-\mathrm{D}^{\frac{1}{2}} x} \Gamma+\int_{a}^{x} \mathrm{e}^{\mathrm{D}^{\frac{1}{2}}(x-\tau)} \mathrm{F}(\tau) d \tau\right. \\
& \left.-\int_{0}^{x} \mathrm{e}^{-\mathrm{D}^{\frac{1}{2}}(x-\tau)} \mathrm{G}(\tau) d \tau\right),
\end{aligned}
$$

onde

$$
\begin{aligned}
& \mathrm{F}(x)=\frac{1}{2}\left[\mathrm{X}^{-1} \mathrm{~S}_{\mathrm{I}}(x)+\mathrm{D}^{-\frac{1}{2}} \mathrm{X}^{-1} \mathrm{BS}_{\mathrm{P}}(x)\right], \\
& \mathrm{G}(x)=\frac{1}{2}\left[\mathrm{X}^{-1} \mathrm{~S}_{\mathrm{I}}(x)-\mathrm{D}^{-\frac{1}{2}} \mathrm{X}^{-1} \mathrm{BS}_{\mathrm{P}}(x)\right],
\end{aligned}
$$

e os vetores desconhecidos $\triangle$ e $\Gamma$ são obtidos pela aplicação das condições de contorno (2.2-2.3). Assim, obtemos a solução ELTA $_{N}$ dada pela equação (2.4) nas direções discretas $\mu_{k}$, onde $u\left(x, \mu_{k}\right)$ e $v\left(x, \mu_{k}\right)$ são expressos, respectivamente, pelas componentes das equações matriciais (3.6) e (3.7).

\section{Resultados Numéricos}

Com o objetivo de mostrar a eficiência sob o ponto de vista computacional da formulação ELTA ${ }_{N}$ comparada à da formulação LTA $_{N}$, vamos apresentar simulações numéricas para dois problemas de transporte com diferentes fontes e altas ordens de quadratura. Inicialmente, vamos considerar um problema de transporte numa placa com espessura $a=1$, vácuo em $x=0$ e em $x=1$, com o termo de fonte

$$
\mathrm{S}(x, \mu)=\frac{\sigma_{s}}{2} \sum_{k=0}^{\mathrm{L}} \beta_{k} \mathrm{P}_{k}(\mu) \mathrm{P}_{k}\left(\mu_{0}\right) \mathrm{e}^{-2 x},
$$

espalhamento anisotrópico de grau $\mathrm{L}=299$ com $\beta_{k}$ assumindo os valores considerados em Gonçalves et al. [19], $\sigma_{t}=1$ e $\sigma_{s}=0.95$. Problemas similares a esse aparecem na solução da transferência radiativa em nuvens pelo método de Chandrasekhar $[2,17]$. Na tabela 1 , apresentamos resultados numéricos para as formulações LTA $_{\mathrm{N}}$ e ELTA $\mathrm{N}_{\mathrm{N}}$ para o fluxo escalar em $x=0$ e em $x=1$, com N variando de 150 a 500. Devido ao caráter idealizado deste problema, fizemos uma comparação com os resultados numéricos do método $\mathrm{LTS}_{\mathrm{N}}$, com $\mathrm{N}$ variando de 300 a 1000 , uma vez que sua convergência já foi demonstrada $[24,25]$ e a equivalência entre as formulações $A_{N}$ e $S_{2 N}$ foi estabelecida [15]. Ademais, o método $\operatorname{LTS}_{N}$ foi utilizado com sucesso em uma ampla classe de problemas de transporte $[18,19,26]$. Neste problema, as integrais de convolução provenientes das soluções LTA $_{N}$, ELTA $_{N}$ e LTS $S_{N}$ foram calculadas analiticamente. 
Tabela 1. Resultados numéricos para o fluxo escalar pelos métodos $\mathrm{LTA}_{\mathrm{N}}, \mathrm{ELTA}_{\mathrm{N}}$ e $\operatorname{LTS}_{\mathrm{N}}\left(\mathrm{L}=299, a=1, \sigma_{s}=0.95, \sigma_{t}=1.0\right.$ e termo de fonte dado na equação $(4.1))$.

\begin{tabular}{|c|c|c|c|c|}
\hline Método & $\begin{array}{l}\text { Fluxo escalar } \\
\text { em } x=0\end{array}$ & $\begin{array}{l}\text { Fluxo escalar } \\
\text { em } x=0.5\end{array}$ & $\begin{array}{l}\text { Fluxo escalar } \\
\text { em } x=1\end{array}$ & $\begin{array}{l}\text { Tempo } \\
\text { (s) }\end{array}$ \\
\hline $\mathrm{LTA}_{150}$ & $1.1159321527 \times 10^{-1}$ & $3.8860733962 \times 10^{-1}$ & $3.4840605528 \times 10^{-1}$ & 4.06 \\
\hline $\mathrm{LTA}_{200}$ & $1.1159321527 \times 10^{-1}$ & $3.8860733962 \times 10^{-1}$ & $3.4840605528 \times 10^{-1}$ & 11.28 \\
\hline $\mathrm{LTA}_{250}$ & $1.1159321527 \times 10^{-1}$ & $3.8860733962 \times 10^{-1}$ & $3.4840605528 \times 10^{-1}$ & 21.64 \\
\hline $\mathrm{LTA}_{300}$ & $1.1159321527 \times 10^{-1}$ & $3.8860733962 \times 10^{-1}$ & $3.4840605528 \times 10^{-1}$ & 37.36 \\
\hline $\mathrm{LTA}_{350}$ & $1.1159321527 \times 10^{-1}$ & $3.8860733962 \times 10^{-1}$ & $3.4840605528 \times 10^{-1}$ & 61.46 \\
\hline $\mathrm{LTA}_{400}$ & $1.1159321527 \times 10^{-1}$ & $3.8860733962 \times 10^{-1}$ & $3.4840605528 \times 10^{-1}$ & 103.82 \\
\hline $\mathrm{LTA}_{450}$ & $1.1159321527 \times 10^{-1}$ & $3.8860733962 \times 10^{-1}$ & $3.4840605528 \times 10^{-1}$ & 132.85 \\
\hline LTA $_{500}$ & $1.1159321527 \times 10^{-1}$ & $3.8860733962 \times 10^{-1}$ & $3.4840605528 \times 10^{-1}$ & 179.32 \\
\hline$\overline{\text { ELTA }}_{150}$ & $1.1159321368 \times 10^{-1}$ & $3.8860733783 \times 10^{-1}$ & $3.4840605357 \times 10^{-1}$ & 0.66 \\
\hline ELTA $_{200}$ & $1.1159321974 \times 10^{-1}$ & $3.8860734466 \times 10^{-1}$ & $3.4840605999 \times 10^{-1}$ & 1.53 \\
\hline $\mathrm{ELTA}_{250}$ & $1.1159321863 \times 10^{-1}$ & $3.8860734324 \times 10^{-1}$ & $3.4840605960 \times 10^{-1}$ & 3.19 \\
\hline ELTA $_{300}$ & $1.1159318481 \times 10^{-1}$ & $3.8860730624 \times 10^{-1}$ & $3.4840601982 \times 10^{-1}$ & 5.28 \\
\hline ELTA $_{350}$ & $1.1159316634 \times 10^{-1}$ & $3.8860728572 \times 10^{-1}$ & $3.4840599869 \times 10^{-1}$ & 8.33 \\
\hline ELTA $_{400}$ & $1.1159318821 \times 10^{-1}$ & $3.8860730976 \times 10^{-1}$ & $3.4840602446 \times 10^{-1}$ & 13.14 \\
\hline ELTA $_{450}$ & $1.1159319019 \times 10^{-1}$ & $3.8860731162 \times 10^{-1}$ & $3.4840602652 \times 10^{-1}$ & 17.47 \\
\hline ELTA $_{500}$ & $1.1159343808 \times 10^{-1}$ & $3.8860758817 \times 10^{-1}$ & $3.4840629830 \times 10^{-1}$ & 23.49 \\
\hline $\operatorname{LTS}_{300}$ & $1.1159794477 \times 10^{-1}$ & $3.8860571600 \times 10^{-1}$ & $3.4841194710 \times 10^{-1}$ & 5.55 \\
\hline $\mathrm{LTS}_{400}$ & $1.1159580545 \times 10^{-1}$ & $3.8860644424 \times 10^{-1}$ & $3.4840929142 \times 10^{-1}$ & 13.67 \\
\hline $\operatorname{LTS}_{500}$ & $1.1159484851 \times 10^{-1}$ & $3.8860677330 \times 10^{-1}$ & $3.4840809848 \times 10^{-1}$ & 24.66 \\
\hline $\operatorname{LTS}_{600}$ & $1.1159433878 \times 10^{-1}$ & $3.8860694941 \times 10^{-1}$ & $3.4840746178 \times 10^{-1}$ & 41.54 \\
\hline $\operatorname{LTS}_{700}$ & $1.1159403532 \times 10^{-1}$ & $3.8860705453 \times 10^{-1}$ & $3.4840708231 \times 10^{-1}$ & 67.41 \\
\hline $\operatorname{LTS}_{800}$ & $1.1159384012 \times 10^{-1}$ & $3.8860712225 \times 10^{-1}$ & $3.4840683804 \times 10^{-1}$ & 110.48 \\
\hline LTS $_{900}$ & $1.1159370717 \times 10^{-1}$ & $3.8860716843 \times 10^{-1}$ & $3.4840667161 \times 10^{-1}$ & 143.20 \\
\hline $\mathrm{LTS}_{1000}$ & $1.1159361256 \times 10^{-1}$ & $3.8860720131 \times 10^{-1}$ & $3.4840655313 \times 10^{-1}$ & 191.69 \\
\hline
\end{tabular}

O segundo problema de transporte em uma placa a ser considerado apresenta os mesmos dados e condições de contorno do problema anterior, exceto pelo tipo de fonte utilizada, nesse caso uma gaussiana

$$
\mathrm{S}(x, \mu)=\mathrm{e}^{0.5(\mathrm{x}-0.5)^{2}} .
$$

$\mathrm{Na}$ tabela 2, apresentamos resultados numéricos para as formulações $\mathrm{LTA}_{\mathrm{N}} \mathrm{e}$ ELTA $_{\mathrm{N}}$ para o fluxo escalar em $x=0, x=0.5$ e em $x=1$, com $\mathrm{N}$ variando de 150 a 500. Também apresentamos comparações numéricas com os resultados obtidos pelo método $\mathrm{LTS}_{\mathrm{N}}$, com N variando de 300 a 1000, pelas mesmas razões expostas acima. Aqui, as integrais de convolução foram calculadas numericamente usando quadratura gaussiana com 50 pontos. 
Tabela 2. Resultados numéricos para o fluxo escalar pelos métodos $\mathrm{LTA}_{\mathrm{N}}, \mathrm{ELTA}_{\mathrm{N}}$ e $\operatorname{LTS}_{\mathrm{N}}\left(\mathrm{L}=299, a=1, \sigma_{s}=0.95, \sigma_{t}=1.0\right.$ e termo de fonte dado na equação $(4.2))$.

\begin{tabular}{||l|l|l|l|c||}
\hline Método & $\begin{array}{l}\text { Fluxo esca- } \\
\text { lar em x=0 }\end{array}$ & $\begin{array}{l}\text { Fluxo es- } \\
\text { calar } \\
\text { x=0.5 }\end{array}$ & $\begin{array}{l}\text { Fluxo esca- } \\
\text { lar em x=1 }\end{array}$ & Tempo (s) \\
\hline \hline LTA $_{150}$ & 1.2260309145 & 1.7033491796 & 1.2260309145 & 2.66 \\
\hline LTA $_{200}$ & 1.2260271537 & 1.7033734812 & 1.2260271537 & 7.55 \\
\hline LTA $_{250}$ & 1.2260259146 & 1.7033736206 & 1.2260259146 & 13.58 \\
\hline LTA $_{300}$ & 1.2260264917 & 1.7033708057 & 1.2260264917 & 23.42 \\
\hline LTA $_{350}$ & 1.2260265481 & 1.7033703702 & 1.2260265481 & 38.63 \\
\hline LTA $_{400}$ & 1.2260264719 & 1.7033707669 & 1.2260264719 & 68.79 \\
\hline LTA $_{450}$ & 1.2260264659 & 1.7033709661 & 1.2260264659 & 83.65 \\
\hline LTA $_{500}$ & 1.2260264770 & 1.7033709468 & 1.2260264770 & 113.34 \\
\hline \hline ELTA $_{150}$ & 1.2260309046 & 1.7033491689 & 1.2260309046 & 0.77 \\
\hline ELTA $_{200}$ & 1.2260271795 & 1.7033735094 & 1.2260271795 & 1.91 \\
\hline ELTA $_{250}$ & 1.2260259364 & 1.7033736413 & 1.2260259364 & 4.14 \\
\hline ELTA $_{300}$ & 1.2260263037 & 1.7033706152 & 1.2260263037 & 6.92 \\
\hline ELTA $_{350}$ & 1.2260262469 & 1.7033700622 & 1.2260262469 & 10.95 \\
\hline LLTA $_{400}$ & 1.2260263067 & 1.7033705958 & 1.2260263067 & 17.05 \\
\hline ELTA $_{450}$ & 1.2260263090 & 1.7033708025 & 1.2260263090 & 23.13 \\
\hline ELTA $_{500}$ & 1.2260278055 & 1.7033723635 & 1.2260278055 & 31.52 \\
\hline \hline LTS $_{300}$ & 1.2262043399 & 1.7034827849 & 1.2262043399 & 3.56 \\
\hline LTS $_{400}$ & 1.2261869724 & 1.7034881057 & 1.2261869724 & 9.14 \\
\hline LTS $_{500}$ & 1.2261791862 & 1.7034905099 & 1.2261791862 & 16.39 \\
\hline LTS $_{600}$ & 1.2261750342 & 1.7034917966 & 1.2261750342 & 27.13 \\
\hline LTS $_{700}$ & 1.2261725610 & 1.7034925646 & 1.2261725610 & 44.33 \\
\hline LTS $_{800}$ & 1.2261709694 & 1.7034930594 & 1.2261709694 & 74.50 \\
\hline LTS $_{900}$ & 1.2261698848 & 1.7034933968 & 1.2261698848 & 92.91 \\
\hline LTS $_{1000}$ & 1.2261691114 & 1.7034936371 & 1.2261691114 & 123.01 \\
\hline
\end{tabular}

Analisando os resultados obtidos, podemos verificar que o tempo de processamento, quando se faz uso da formulação ELTA $_{N}$, é bem menor que o tempo demandado com a utilização da formulação $\mathrm{LTA}_{\mathrm{N}}$. Essa constatação já havia sido feita quando os dois métodos foram utilizados na resolução de problemas homogêneos de transporte [5]. Ademais, podemos observar uma coincidência de quatro algarítimos significativos entre as formulações e que dentre as três formulações estudadas, a do método $\operatorname{LTS}_{\mathrm{N}}$ foi a que apresentou o pior desempenho, com uma convergência mais lenta e maior esforço computacional.

No nosso conhecimento, a construção de uma solução particular para uma fonte arbitrária e altos graus de anisotropia no método $\mathrm{LTA}_{\mathrm{N}}$ é uma novidade proposta por Cardona et al. [4, 9]. Convém ressaltar que o método dos coeficientes a determinar [20], utilizado para encontrar soluções particulares, é aplicado apenas para 
algumas fontes especiais. Embora utilizamos uma fonte exponencial no primeiro problema e uma fonte gaussiana no segundo, é oportuno salientar que poderiamos ter utilizado diversos tipos de fontes, desde que as integrais de convolução tenham solução. E mais, na construção de soluções particulares pelo Método das Funções de Green $[1,10,12]$ necessita-se da construção da função de Green para cada condição de contorno, enquanto que na técnica da transformada de Laplace, constroí-se uma única solução, a qual é válida para qualquer tipo de condição de contorno. A influência destas condições aparece em constantes de integração arbitrárias, as quais são obtidas do sistema algébrico linear resultante da aplicação de uma condição específica.

Finalizando, podemos dizer que o objetivo deste trabalho foi alcançado, uma vez que o método proposto apresenta um significativo ganho no tempo de processamento sem implicar numa maior complexidade na elaboração dos códigos computacionais. Dessa forma, caminhamos na direção de resolver problemas de transporte em uma placa, em tempo real.

Como trabalho futuro, pretendemos resolver problemas de transferência radiativa em nuvens e problemas transientes de transporte pelo método exposto neste artigo.

Abstract. Recently a new version of the $\mathrm{LTA}_{\mathrm{N}}$ method was presented to solve homogeneous linear transport problems in a slab. This approach was called ELTA method and it is based on the diagonalization of a $\mathrm{N} \times \mathrm{N}$ matrix, instead of a $(2 \mathrm{~N}) \times(2 \mathrm{~N})$ matrix as in the original $\mathrm{LTA}_{\mathrm{N}}$ method. In this work, we extend this formulation to solve non-homogeneous transport problems. Numerical simulations and comparisons with the $\mathrm{LTS}_{\mathrm{N}}$ and $\mathrm{LTA}_{\mathrm{N}}$ methods are presented.

\section{Referências}

[1] L.B. Barichello, R.D.M. Garcia, C.E. Siewert, Particular solutions for the discrete ordinates method. Journal of Quantitative Spectroscopy and Radiative Transfer, 64 (2000), 219-266.

[2] J.D. Brancher, M.T. Vilhena, C.F. Segatto, The $\operatorname{LTS}_{N}$ Solution for radiative transfer problem without azimuthal symmetry with severe anisotropy, Journal of Quantitative Spectroscopy and Radiative Transfer, 62 (1999), 743-753.

[3] A.V. Cardona, "Um Método Genérico de Solução Analítica para algumas Aproximações da Equação Linear de Transporte", Tese de Doutorado, Engenharia Mecânica, UFRGS, Porto Alegre, RS, 1996.

[4] A.V. Cardona, R.P. Pazos, M.T.M.B. Vilhena, The state-of-the-art of the LTA $_{\mathrm{N}}$ method, in "Icone $12-12^{\text {th }}$ International Conference on Nuclear Energy", Proceeding of $12^{\text {th }}$ International Conference on Nuclear Energy, Arlington, Virginia (EUA), ASME, 2004.

[5] A.V. Cardona, R. Vasques, M.T.M.B. Vilhena, Uma nova versão do método LTA $_{N}$, TEMA Tend. Mat. Apl. Comput., 5, No. 1, (2004), 49-54. 
[6] A.V. Cardona, M.T. Vilhena, A comparative study of analytical solutions for some one-dimensional transport equation approximations, Progress in Nuclear Energy, 33 (1998), 289-300.

[7] A.V. Cardona, M.T.M.B. Vilhena, Analytical solution for the $\mathrm{A}_{\mathrm{N}}$ approximation, Annals of Nuclear Energy, 24 (1997), 495-505.

[8] A.V. Cardona, M.T.M.B. Vilhena, Solução analítica da aproximação $A_{N}$ da equação de transporte linear com simetria planar, em "Anais do X Encontro de Física de Reatores e Termo-Hidráulica", pp. 528-531, Águas de Lindóia, SP, 1995.

[9] A.V. Cardona, M.T.M.B. Vilhena, J.V.P. de Oliveira, R. Vasques, The onedimensional $\mathrm{LTA}_{\mathrm{N}}$ solution in a Slab with high order of quadrature, in "Proceedings of 18th International Conference on Transport Theory", pp. 260-264, Rio de Janeiro, Brazil, 2003.

[10] R. Ciolini, G. Coppa, B. Montagnini, P. Ravetto, Simplified $P_{N}$ and $A_{N}$ methods in neutron transport, Progress in Nuclear Energy, 40 (2002), 237264 .

[11] G. Coppa, P. Ravetto, An approximate method to study the one velocity neutron integral transport equation, Annals of Nuclear Energy, 9 (1982), 169-174.

[12] G. Coppa, P. Ravetto, M. Sumini, The $A_{N}$ method in monokinetic transport theory: convergence and numerical approximations, Transport Theory and Statistical Physics, 10 (1981), 161-181.

[13] G. Coppa, P. Ravetto, M. Sumini, Approximate solution to neutron transport equation with linear anisotropic scattering, Journal of Nuclear Science and Technology, 20 (1983), 822-831.

[14] G. Coppa, P. Ravetto, M. Sumini, The $A_{N}$ Method and the spherical harmonics approximation in neutron transport theory, Annals of Nuclear Energy, 9 (1982), 435-437.

[15] G. Coppa, P. Ravetto, M. Sumini, Numerical performance of the $A_{N}$ method and comparisons with $\mathrm{S}_{\mathrm{N}}$ calculations, Atomkernenergie Kerntechnik, 42 (1983), 107-110.

[16] J. Duderstadt, W.R. Martin, "Transport Theory", John Wiley \& Sons, Inc., New York, 1979.

[17] R.D.M. Garcia, C.E. Siewert, Benchmark Results in radiative transfer, Transport Theory and Statistical Physics, 14 (1985), 437-483.

[18] G.A. Gonçalves, G. Orengo, M.T. Vilhena, C.O. Graça, LTS $_{N}$ Solution of the adjoint neutron transport equation with arbitrary source for high order of quadrature in a homogeneous Slab, Annals of Nuclear Energy, 29 (2002), $561-569$. 
[19] G.A. Gonçalves, C.F. Segatto, M.T. Vilhena, The LTS $_{N}$ particular solution in a Slab for an srbitrary source and large order of quadrature, Journal of Quantitative Spectroscopy and Radiative Transfer, 66 (2000), 271-276.

[20] W. Kaplan, "Ordinary Equations, Addison-Wesley: Reading", Massachusetts, 1967.

[21] J. Lenoble, "Standard Procedures to Compute Atmospheric Radiative Transfer in a Scattering Atmosphere", NCAR, Boulder, 1977.

[22] E.E. Lewis, F.W. Miller Jr., "Computational Methods of Neutron Transport", American Nuclear Society, Illinois, 1993.

[23] G.J. Marchuk, "Methods of Numerical Mathematics", Springer-Verlag, New York, 1975.

[24] R.P. Pazos, M. Thompson, M.T.M.B. Vilhena, Error bounds for spectral collocation method for the linear Boltzmann equation, International Journal of Computation and Numerical Analysis and Application, 1 (2002), 237-268.

[25] R.P. Pazos, M.T.M.B. Vilhena, Convergence in transport theory, Applied Numerical Mathematics, 30 (1999), 79-92.

[26] C.F. Segatto, M.T. Vilhena, The State-of-the-art of the LTS $_{N}$ Method, in "Proceedings of The International Conference on Mathematics and Computation, Reactor Physics and Environmental Analysis in Nuclear Applications", Vol. 2, pp. 1618-1631, Madrid, Spain, 1999.

[27] C.F. Segatto, M.T.M.B. Vilhena, M.G. Gomes, The one-dimensional LTS $_{N}$ solution in a Slab with high degree of quadrature, Annals of Nuclear Energy, 26 (1999), 924-925.

[28] Z. Trzaska, An efficient algorithm for partial fraction expansion of the linear matrix pencil inverse, Journal of the Franklin Institute, 324 (1987), 465-477.

[29] M.T. Vilhena, L.B. Barichello, A new analytical approach to solve the neutron transport equation, Kerntechnik, 56 (1991), 334-336.

[30] M.T. Vilhena, L.B. Barichello, General approach to one-group one-dimensional transport equation, Kerntechnik, 58 (1993), 182-184. 